Proyecciones Journal of Mathematics

Vol. 25, No2, pp. 151-178, August 2006.

Universidad Católica del Norte

Antofagasta - Chile

\title{
DIFFEOLOGICAL SPACES
}

\author{
MARTIN LAUBINGER \\ LOUISIANA STATE UNIVERSITY, LOUISIANA - U.S.A.
}

Received : January 2006. Accepted : April 2006

\begin{abstract}
We define diffeological spaces and give some examples. The diffeological category contains the category of smooth manifolds as full subcategory. We prove that diffeological spaces and smooth maps form a cartesian closed category.

The concepts of differential form and tangent functor are extended from smooth manifolds to diffeological spaces.
\end{abstract}

Subjclass : 57R55 Differentiable Structures. See www.ams.org/msc 


\section{Introduction}

Smooth $n$-dimensional manifolds are by definition topological spaces which locally look like $\mathbf{R}^{n}$ and exhibit smooth coordinate changes.

In contrast, diffeological spaces do not a priori carry a topology or a dimension. The central concept is that of 'smooth maps'. So we define diffeological spaces via a collection of maps from open sets of $\mathbf{R}^{n}$ for all $n$ into a set, such that this collection is closed under smooth coordinate changes. This will lead to a concept which is better from a categorical point of view: diffeological spaces form a cartesian closed category, which we will prove in Chapter 3.

Even though the concept of a diffeological space is very general, we can still extend some concepts from differential geometry to this new setting. For example, in Chapter 4 we define differential forms, and in Chapter 5 we define a tangent functor. Smooth manifolds are an example of diffeological spaces, and in the last chapter we show that the new definitions agree with the classical ones on the subcategory of manifolds.

We start by fixing the notation for the category of open subsets of real spaces and smooth maps.

Definition 1.1. Let OR be the category whose objects are all open sets of all $\mathbf{R}^{n}, n \in \mathbf{N}$, and whose morphisms are all smooth maps between these open sets. The collection of smooth maps with domain in $\mathbf{R}^{n}$ and range in $\mathbf{R}^{p}$ will be denoted by $\mathcal{L}^{n p}$. The collection of maps from an open set $U \subset \mathbf{R}^{n}$ into a set $X$ is denoted by $\mathcal{L}^{n}(X)$, and we will use the letters $\alpha, \beta, \gamma, \ldots$ for elements of $\mathcal{L}^{n}(X)$. The corresponding domains are denoted by $U_{\alpha}, U_{\beta}, U_{\gamma}$ respectively. Lastly, let $\mathcal{L}(X)$ be the union of all the $\mathcal{L}^{n}(X)$.

Definition 1.2. Given maps $\alpha \in \mathcal{L}^{n p}$ and $\beta \in \mathcal{L}^{p}(X)$, we say that $(\beta, \alpha)$ is a composable pair if $\alpha\left(U_{\alpha}\right) \subseteq U_{\beta}$. We will also just say that $\alpha$ and $\beta$ are composable. A collection $\left\{\alpha_{i} \mid i \in I\right\} \subset \mathcal{L}^{n}(X)$ is called compatible if $x \in U_{i} \cap U_{j}$ implies $\alpha_{i}(x)=\alpha_{j}(x)$. Every compatible family has a unique smallest extension $\alpha$ which has domain $\cup_{i} U_{i}$ and is defined to be equal to $\alpha_{i}$ on $U_{i}$. 
Now we proceed to define diffeological spaces and smooth maps between diffeological spaces as in [Sou85].

Definition 1.3 (Diffeology). Given a set $X$ consider a collection $\mathcal{P}(X)=$ $\cup_{n} \mathcal{P}^{n}(X)$ of maps into $X$, such that $\mathcal{P}^{n}(X) \subset \mathcal{L}^{n}(X)$. We call $\mathcal{P}(X)$ a diffeology if it satisfies the following axioms:

(D1) Every constant map $x: U \rightarrow X$ is in $\mathcal{P}(X)$.

(D2) Given a compatible family of maps in $\mathcal{P}^{n}(X)$, its smallest extension is again in $\mathcal{P}^{n}(X)$.

(D3) If $h \in \mathcal{L}^{p n}$ and $\alpha \in \mathcal{P}^{n}(X)$ are composable, then $\alpha \circ h \in \mathcal{P}^{p}(X)$.

The elements of $\mathcal{P}^{n}(X)$ are called n-plots or simply plots of the diffeology. The pair $(X, \mathcal{P}(X))$ is called a diffeological space.

Definition 1.4 (D-topology). Given a diffeological space $(X, \mathcal{P}(X))$ we define the -topology to be the initial topology with respect to the plots. That is, a subset $U \subset X$ is open if and only if for each plot $\alpha \in \mathcal{P}(X)$, the inverse image $\alpha^{-1}(U)$ is open in $U_{\alpha}$.

We can immediately give two examples.

Example 1.5 (Indiscrete Diffeology). Given any set $X$, the collection $\mathcal{L}(X)$ of all possible maps into $X$ with open domain is a diffeology. The only open sets are $X$ and the empty set, so the -topology is the indiscrete topology.

Example 1.6 (Discrete Diffeology). Let $\mathcal{P}(X)$ denote the collection of all locally constant maps. This is the smallest possible diffeology on the set $X$. Note that we cannot restrict to all constant maps! This would violate (D2): If we take two maps that are constant, taking distinct values on disjoint domains, their smallest extension is merely locally constant. Every subset of $X$ is open in the -topology, so $X$ is a discrete topological space. 
Axiom (D2) has an immediate consequence, which says that being a plot is a local property of a map.

Lemma 1.7. Let $(X, \mathcal{P}(X))$ be a diffeological space, $U_{\alpha} \subset \mathbf{R}^{n}$ open and $\alpha: U_{\alpha} \rightarrow X$ a map. If for each $x \in U_{\alpha}$ there is a neighborhood $V$ of $x$ in $U_{\alpha}$ such that $\alpha$ restricted to $V$ is a plot, then $\alpha$ is a plot.

Proof. Given $x \in U_{\alpha}$, let $V_{x}$ be a neighborhood of $x$ such that $\alpha_{x}:=\left.\alpha\right|_{V_{x}}$ is a plot. Then $\left\{\alpha_{x} \mid x \in U_{\alpha}\right\}$ is a compatible family of plots with smallest extension $\alpha$. So it follows from axiom (D2) that $\alpha$ is a plot.

Lemma 1.8. The -topology of a diffeological space $(X, \mathcal{P}(X))$ is locally arc-connected.

Proof. Given a point $x \in X$, let $P$ be the arc-component of $x$. So for each $y \in P$, there is a continuous path from $x$ to $y$. It suffices to show that $P$ is an open set. Let $\alpha: U_{\alpha}$ rightarrow $X$ be a plot, and suppose that $\alpha^{-1}(P)$ is nonempty, containing a point $p$. As $U_{\alpha}$ is open, we can find an open ball $B \subset U_{\alpha}$ containing $p$. We want to show $B \subset \alpha^{-1}(P)$, so let $q \in B$. To show that $\alpha(q) \in P$, we have to connect $x$ and $\alpha(q)$ with a continuous path. By choice of $p$, the image $\alpha(p)$ can be connected to $x$ by a continuous path. Now $p$ and $q$ can be connected in $B$, and we can compose this path with $\alpha$ to get a path from $\alpha(p)$ to $\alpha(q)$. Then by concatenation, we get a path from $x$ to $\alpha(q)$. Thus $\alpha(q) \in P$. This proves $B \subset \alpha^{-1}(P)$, hence $\alpha^{-1}(P)$ is open in $U_{\alpha}$, which concludes the proof.

Intersections of diffeologies are again diffeologies, and therefore it is easy to define the diffeology generated by a given family of functions. We will characterize the plots of such diffeologies.

Lemma 1.9. Given a family $\left\{\mathcal{P}_{i} \mid i \in I\right\}$ of diffeologies on a set $X$, the intersection $\cap_{i} \mathcal{P}_{i}$ is again a diffeology. 
Proof. We have to verify the three axioms (D1)-(D3) for $\mathcal{P}:=\cap_{i} \mathcal{P}_{i}$. By definition, each of the diffeologies $\mathcal{P}_{i}$ contains all constant maps, thus the same is true for their intersection. Every compatible family of $n$-plots in $\mathcal{P}$ is also compatible in each of the $\mathcal{P}_{i}$. Therefore the smallest common extension is element of each $\mathcal{P}_{i}$, hence also of $\mathcal{P}$. Similarly, if $\alpha \in \mathcal{P}$ and $h$ are composable, then $\alpha \circ h \in \mathcal{P}_{i}$ for all $i \in I$, and therefore also $\alpha \circ h \in \mathcal{P}$.

Corollary 1.10. If $\mathbf{F} \subset \mathcal{L}(X)$ is a collection of maps into $X$, then there is a unique smallest diffeology on $X$ containing $\mathbf{F}$. We will denote this diffeology by $\langle\mathcal{F}\rangle$

Proof. Note that the indiscrete diffeology contains F. So the collection of all diffeologies on $X$ which contain $\mathbf{F}$ is nonempty, and we can take its intersection.

Definition 1.11. If $\mathcal{P}(X)$ is the smallest diffeology containing a collection of maps $\mathbf{F}$, then the collection is called a generating family for the diffeology. For a given generating family $\mathbf{F}$, let $n_{\mathbf{F}}$ denote the supremum

$$
n_{\mathbf{F}}:=\sup \left\{\operatorname{dim}\left(U_{\alpha}\right) \mid \alpha \in \mathbf{F}\right\} .
$$

Then the dimension of a diffeological space $(X, \mathcal{P}(X))$ is the infimum

$$
\operatorname{dim}(X, \mathcal{P}(X)):=\inf \left\{n_{\mathbf{F}} \mid \mathbf{F} \text { generates } \mathcal{P}(X)\right\}
$$

Lemma 1.12. Let $X$ be a set and $\mathbf{F} \subset \mathcal{L}(X)$. Then a map $\alpha: U_{\alpha} \rightarrow X$ is in $\langle\mathbf{F}\rangle$ if and only if it satisfies

(G) For each point $x \in U_{\alpha}$ there is an open neighborhood $V \subset U_{\alpha}$ of $x$ such that the restriction of $\alpha$ to $V$ is either constant or of the form $f \circ h$ for some $f \in \mathbf{F}$ and a smooth map $h: V \rightarrow U_{f}$. 
Proof. Let $\mathcal{P}$ denote the collection of maps $\alpha$ satisfying (G). Clearly, $\mathcal{P}$ contains $\mathbf{F}$. We will first show that $\mathcal{P}$ is a diffeology. Constant maps certainly satisfy (G), therefore (D1) holds. Now take a compatible family $\alpha_{i} \in \mathcal{P}$ with smallest extension $\alpha$. Each $x \in U_{\alpha}$ is contained in some $\operatorname{dom}\left(\alpha_{i}\right)=U_{i}$ and has therefore a neighborhood in $U_{i}$ on which $\alpha$ is constant or of the form $f \circ g$ with smooth $g$ and $f \in \mathbf{F}$. Therefore $\alpha$ is in $\mathcal{P}$, which shows (D2). To verify (D3), let $\alpha$ and $h: U \rightarrow U_{\alpha}$ be composable, where $\alpha$ is in $\mathcal{P}$ and $h$ is smooth. Let $x \in U$ and $y=h(x)$. Then there is a neighborhood $V$ of $y$ such that either

- $\left.\alpha\right|_{V}$ is constant. Then $\left.\alpha \circ h\right|_{h^{-1}(V)}$ is also constant.

or

- $\left.\alpha\right|_{V}$ is of the form $f \circ g$ for smooth $g$ and $f \in \mathcal{P}$. Then $\left.\alpha \circ h\right|_{h^{-1}(V)}=f \circ g \circ h$.

As $h^{-1}(V)$ is an open neighborhood of $x$, in either case we have that $\alpha \circ h \in \mathcal{P}$. This proves (D3). So $\mathcal{P}$ is a diffeology containing $\mathbf{F}$. If we can show $\mathcal{P} \subset\langle\mathbf{F}\rangle$, we can conclude equality as $\langle\mathbf{F}\rangle$ is minimal containing $\mathbf{F}$. Let $\alpha \in \mathcal{P}$. Then each point $x \in U_{\alpha}$ has a neighborhood $V_{x}$ such that $\alpha_{x}:=\left.\alpha\right|_{V_{x}}$ is either constant or of the form $f \circ g$ with smooth $g$ and $f \in \mathbf{F}$. So in either case, $\alpha_{x}$ is in $\langle\mathbf{F}\rangle$, and the collection of the $\alpha_{x}$ is a compatible family with smallest extension $\alpha$. Thus $\alpha \in\langle\mathbf{F}\rangle$, which concludes the proof.

Diffeological spaces will be the objects of a category. Let us now define the morphisms.

Definition 1.13 (Smooth Maps). Given two diffeological spaces $(X, \mathcal{P}(X))$ and $(Y, \mathcal{P}(Y))$ and a map $f: X \rightarrow Y$, we say that $f$ is smooth if for every $\alpha \in \mathcal{P}(X)$, the composition $f \circ \alpha$ is in $\mathcal{P}(Y)$.

Lemma 1.14. If the diffeology $\mathcal{P}(X)$ is generated by a family $\mathbf{F}$ of functions, then a map $f: X \rightarrow Y$ is smooth if $f \circ \alpha \in \mathcal{P}(Y)$ for all $\alpha \in \mathbf{F}$.

Proof. Let $\alpha$ be a plot for $X$, and $x \in U_{\alpha}$. By Lemma 1.12 we can choose an open neighborhood $U_{x}$ of $x$ in $U_{\alpha}$ such that $\left.\alpha\right|_{U}$ is either constant or of the form $\beta \circ h$ for some $\beta \in \mathbf{F}$ and a smooth map $h$. In the first case $\left.f \circ \alpha\right|_{U}$ is constant and thus in $\mathcal{P}(Y)$. In the second case, $\left.f \circ \alpha\right|_{U}=f \circ \beta \circ h$ which is in $\mathcal{P}(Y)$ since by assumption, $f \circ \beta \in \mathcal{P}(Y)$. The open sets $U_{x}$ cover $U_{\alpha}$, so we use axiom (D2) to conclude that $f \circ \alpha \in \mathcal{P}(Y)$, hence $f$ is smooth. 
Definition 1.15 (Diffeological Category). Diffeological spaces as objects and smooth maps as morphisms form a category. We will call this category the diffeological category and denote it by Diff. The collection of morphisms between $(X, \mathcal{P}(X))$ and $(Y, \mathcal{P}(Y))$ will be denoted by $[X, Y]$.

Remark 1.16. The author of [Gio03] denotes this construction as the cartesian closure of the model category $\mathbf{O R}$. He more generally investigates open sets and $C^{n}$-maps as a model category.

\section{Some Examples}

It turns out that diffeological spaces generalize smooth manifolds. Every smooth manifold carries a natural diffeology which we define in Subsection 2.1. But the strength of the generality shows up when we equip mapping spaces with a diffeology in Example 2.10.

\subsection{Manifolds}

Let $M$ be a finite dimensional smooth manifold. The $n$-plots are the maps $\alpha: U_{\alpha} \rightarrow M$ which are smooth in the usual sense of differential geometry. We will briefly check if the three axioms of a diffeology are satisfied. (D1) Clearly, constant maps are smooth. (D2) Let $\alpha$ be the smallest extension of the $\alpha_{i}$. Then given $x \in U_{\alpha}$, there is an $i \in I$ such that $x \in U_{i}$ and hence $\left.\alpha\right|_{U_{i}}=\alpha_{i}$ is smooth. Now smoothness is a local condition, so $\alpha$ is smooth on all of $U_{\alpha}$. (D3) Compositions of smooth maps are smooth.

Lemma 2.1. Given two smooth manifolds $M$ and $N$, the usual smooth maps coincide with the smooth maps in the diffeological sense. In other words, $C^{\infty}(M, N)=[M, N]$.

Proof. The inclusion $C^{\infty}(M, N) \subset[M, N]$ is a consequence of the chain rule, because if $\alpha: U_{\alpha} \rightarrow M$ is a smooth map and $f \in C^{\infty}(M, N)$, then the composition $f \circ \alpha$ is again smooth and hence in $\mathcal{P}(N)$. Conversely, let $f \in[M, N]$. We have to show that $f$ is smooth, so let $\phi: U \rightarrow \mathbf{R}^{n}$ be a chart in a given atlas $\mathbf{A}$ of $M$. By choice of $f$, the map $f \circ \phi^{-1}$ is a plot for $N$, and therefore a smooth map. This is true for every chart in $\mathbf{A}$, which shows that $f \in C^{\infty}(M, N)$. 
Remark 2.2. In the terminology of category theory, the equality $C^{\infty}(M, N)=[M, N]$ is expressed by saying that the category of smooth manifolds is a full subcategory of Diff.

Lemma 2.3. Let $M$ be a $n$-dimensional smooth manifold with atlas $\mathbf{A}$ and the diffeology $\mathcal{P}(M)$ as described above. Then the family $\mathbf{A}^{-1}=\left\{\phi^{-1} \mid \phi \in\right.$ A) generates $\mathcal{P}(M)$.

Proof. Clearly $\left\langle\mathbf{A}^{-1}\right\rangle \subset \mathcal{P}(M)$ as the inverse charts $\phi^{-1}$ are smooth. For the other inclusion, we use the characterization $(G)$ of a generated diffeology from Lemma 1.12. Let $\alpha: U_{\alpha} \rightarrow M$ be a smooth map, $x \in U_{\alpha}$ and let $\phi$ : $U \rightarrow \mathbf{R}^{n}$ be a chart about $\alpha(x)$. Then $V=\alpha^{-1}(U)$ is an open neighborhood of $x$ in $U_{\alpha}$. The map $h:=\left.\phi \circ \alpha\right|_{V}$ is smooth and we can write $\left.\alpha\right|_{V}=\phi^{-1} \circ h$, where $\phi^{-1} \in \mathbf{A}^{-1}$ and $h$ is smooth. So by Lemma 1.12 it follows that $\alpha \in\left\langle\mathbf{A}^{-1}\right\rangle$.

Corollary 2.4. Given a smooth $n$-dimensional manifold, its dimension as a diffeological space (Definition 1.11) is also $n$.

Proof. Lemma 2.3 immediately yields that the diffeological dimension is at most $n$. Now if it were strictly less than $n$, the diffeology would be generated by its collection of $n-1$-plots. But then by Lemma 1.12, every smooth map into $M$ would locally be constant or factor through an open subset of $\mathbf{R}^{n-1}$. This is certainly not true for local diffeomorphisms, e.g. inverse maps of coordinate charts.

The next lemma will characterize the diffeological spaces which are manifolds.

Lemma 2.5. Let $(X, \mathcal{P}(X))$ be a diffeological space, equipped with the $D$ topology. The following are equivalent.

1. There is a smooth atlas $\mathbf{A}$ making $X$ a smooth $n$-dimensional manifold, such that $\mathcal{P}(X)$ is the corresponding manifold diffeology.

2. There is an open cover $\left\{U_{i}\right\}_{i \in I}$ of $X$ and an $n \in \mathbf{N}$ such that for each $i \in I$ there is a diffeomorphism $\phi_{i}$ between $U_{i}$ and some open set $V_{i} \subset \mathbf{R}^{n}$. Here $U_{i}$ is equipped with the subset diffeology, and $V_{i}$ carries the manifold diffeology. 
Proof. First assume that (1) holds, and let $\mathbf{A}=\left\{\left(U_{i}, \phi_{i}\right)\right\}_{i \in I}$. The open sets $U_{i}$ cover $X$, and we claim that the maps $\phi_{i}$ are isomorphisms in Diff. But this is clear, since it is an isomorphism in the category of smooth manifolds, which is a full subcategory of Diff.

Now we assume that $(2)$ holds. Clearly, the pairs $\left(U_{i}, \phi_{i}\right)$ form an atlas, since all compositions $\phi_{i} \circ \phi_{j}^{-1}$ are smooth. Since $\phi_{i}^{-1} \in \mathcal{P}(X)$, the corresponding manifold diffeology is contained in $\mathcal{P}(X)$. To show equality, let $\alpha$ be any plot. But then $\alpha$ is also smooth as a map between manifolds, because for each $i \in I$, the map $\phi_{i}^{-1} \circ \alpha$ is smooth.

\subsection{Generating new diffeological spaces}

The next examples are of a more general nature and allow us to construct new diffeological spaces from old ones. Some of these constructions are familiar from topology and measure theory, for example products and quotients of spaces.

A special feature of the diffeological category is that there is a natural diffeology on the morphism sets. This functional diffeology is used extensively in Chapter 3.

Definition 2.6 (Initial and Final Diffeology). Given a familiy of diffeological spaces $\left\{\left(Y_{i}, \mathcal{P}\left(Y_{i}\right)\right) \mid i \in I\right\}$ and maps $f_{i}: X \rightarrow Y_{i}$ from a set $X$, we define the initial diffeology on $X$ with respect to the maps $f_{i}$ to be the diffeology consisting of all maps $\alpha: U_{\alpha} \rightarrow X$ such that $f_{i} \circ \alpha \in \mathcal{P}\left(Y_{i}\right)$ for all $i \in I$. This is the largest diffeology making all the $f_{i}$ smooth.

Given a family of diffeological spaces $\left\{\left(X_{i}, \mathcal{P}\left(X_{i}\right) \mid i \in I\right\}\right.$ and maps $f_{i}: X_{i} \rightarrow Y$ to a set $Y$, we define the final diffeology on $Y$ with respect to the maps $f_{i}$ to be the diffeology generated by the maps $f_{i} \circ \alpha$ for all $i \in I$ and all plots $\alpha$. It is the smallest diffeology making all the $f_{i}$ smooth.

Definition 2.7. In the above definition, suppose that the family consists of one space only. Let $\mathcal{P}^{f}(X)$ denote the set of all maps $\alpha: U_{\alpha} \rightarrow X$ such that $f \circ \alpha \in \mathcal{P}(Y)$, which is the initial diffeology with respect to $f$. Let $\mathcal{P}_{f}(Y)$ denote the diffeology generated by the $f \circ \alpha$ for $\alpha \in \mathcal{P}(X)$, that is, the final diffeology with respect to $f$. If $f$ is surjective and $\mathcal{P}_{f}(Y)=\mathcal{P}(Y)$ we call $f$ a subduction. If $f$ is injective and $\mathcal{P}^{f}(X)=\mathcal{P}(X)$ we call $f$ an induction. 
Example 2.8 (Subspaces and Quotients). Given a diffeological space $(X, \mathcal{P}(X))$ and a subset $A \subset X$, we obtain a diffeology on $A$ by just taking plots $\alpha \in \mathcal{P}(X)$ which map into $A$. If $\iota: A \rightarrow X$ is the inclusion map, we recognize this diffeology as $\mathcal{P}^{\iota}(A)$. Thus the inclusion map is an induction.

Given a diffeological space $(X, \mathcal{P}(X))$ and an equivalence relation $\sim$ on $X$, we equip $X / \sim$ with the final diffeology with respect to the projection $\pi$. This diffeology makes $\pi$ a subduction.

Example 2.9 (Direct Products and Sums). Given a family of diffeological spaces $\left\{\left(X_{i}, \mathcal{P}\left(X_{i}\right)\right) \mid i \in I\right\}$, we can form disjoint union and direct product of the sets $X_{i}$ in the category of sets and maps. We equip the direct product

$$
\prod_{i} X_{i}
$$

with the initial diffeology with respect to the projections $\pi_{i}$, and the disjoint union

$$
\coprod_{i} X_{i}
$$

with the final diffeology with respect to the injections $\iota_{i}$. Then the projections are subductions and the injections are inductions. Note that for two spaces $X, Y$ the product diffeology is given by plots of the form $\left(\alpha_{1}, \alpha_{2}\right)$ where $\alpha_{1} \in \mathcal{P}(X)$ and $\alpha_{2} \in \mathcal{P}(Y)$.

Example 2.10 (Functional Diffeology). Let two diffeological spaces $(X, \mathcal{P}(X))$ and $(Y, \mathcal{P}(Y))$ be given. Note that an open set $U \subset \mathbf{R}^{n}$ is also a smooth manifold, and hence by Subsection 2.1 also a diffeological space. So by Example 2.9, the product $U \times X$ is a diffeological space. Now we say that $\alpha: U_{\alpha} \rightarrow[X, Y]$ is a plot for $[X, Y]$ if the map $\tilde{\alpha}: U_{\alpha} \times X \rightarrow Y$ defined by $\tilde{\alpha}(u, x)=\alpha(u)(x)$ is smooth. Using the definitions of smooth map and product diffeology, this condition can be reformulated as follows: given $\beta_{1}: U \rightarrow U_{\alpha}$ smooth and $\beta_{2}: U \rightarrow X$ a plot, the map $u \mapsto \alpha\left(\beta_{1}(u)\right)\left(\beta_{2}(u)\right)$ is a plot for $Y$.

Combining the above examples, we get as a very important example the diffeomorphism groups of smooth manifolds. Those groups play a role in areas of applications such as topological fluid dynamics and other parts of theoretical physics (see for example [Don88]). 
Example 2.11 (Diffeomorphism Groups). Let $M$ be a smooth manifold. Then $M$ carries a diffeology by Subsection 2.1, such that

$$
C^{\infty}(M, M)=[M, M] .
$$

So by Example 2.10, the set $C^{\infty}(M, M)$ is a diffeological space, and therefore the subset of $\operatorname{Diff}(M)$ is a diffeological space. Its plots are maps $\alpha: U_{\alpha} \rightarrow$ $\operatorname{Diff}(M)$ such that $\tilde{\alpha}: U_{\alpha} \times M \rightarrow M$ is smooth, where $\tilde{\alpha}$ is defined as in Example 2.10.

\section{Cartesian Closedness}

This section is devoted to the proof of the fact that the category of diffeological spaces is cartesian closed. First we define two functions which are important in this context.

Definition 3.1. Let $X, Y$ and $Z$ be sets. We define the two maps

$$
\Phi: \operatorname{Maps}(X,(Y, Z)) \rightarrow \operatorname{Maps}(X \times Y, Z)
$$

and

$$
\Psi: \operatorname{Maps}(X \times Y, Z) \rightarrow \operatorname{Maps}(X,(Y, Z))
$$

by $\Phi(f)(x, y)=f(x)(y)$ and $\Psi(f)(x)(y)=f(x, y)$. We also write

$$
\tilde{f}:=\Phi(f) \quad \text { and } \quad \bar{f}:=\Psi(f) .
$$

Now let us state the main result of this section.

Theorem 3.2. Let $(X, \mathcal{P}(X)),(Y, \mathcal{P}(Y))$ and $(Z, \mathcal{P}(Z))$ be diffeological spaces. Then $[X \times Y, Z]$ and $[X,[Y, Z]]$ can be equipped with diffeologies if we use the product diffeology for $X \times Y$ and the functional diffeology for the morphism sets. The map $\Psi$ yields an isomorphism

$$
[X \times Y, Z] \cong[X,[Y, Z]]
$$

in the diffeological category, with inverse $\Phi$.

Remark 3.3. This theorem implies that the category Diff is a cartesian closed category. For more details on categories in general, see [Mac71]. Cartesian closed categories generated by maps are treated in [Fro86], and for the concept of cartesian closure we refer to [Gio03]. 
Before we prove this theorem we show that in the diffeological category, evaluation yields a smooth map.

Lemma 3.4. Given diffeological spaces $(X, \mathcal{P}(X))$ and $(Y, \mathcal{P}(Y))$, equip the space $[X, Y] \times X$ with the obvious diffeology. Then the evaluation map

$$
E:[X, Y] \times X \rightarrow Y \quad(f, x) \mapsto f(x)
$$

is smooth.

Proof. Let $\alpha: U_{\alpha} \rightarrow[X, Y] \times X$ be a plot. We write $\alpha$ as $\left(\alpha_{1}, \alpha_{2}\right)$. Then $\alpha_{1}$ is a plot for $[X, Y]$, hence

$$
\tilde{\alpha}_{1}: U_{\alpha} \times X \rightarrow Y
$$

is smooth. Now $\operatorname{E~} \circ \alpha(u)=E\left(\alpha_{1}(u), \alpha_{2}(u)\right)=\alpha_{1}(u)\left(\alpha_{2}(u)\right)$

$=\tilde{\alpha}_{1}\left(u, \alpha_{2}(u)\right)$

$=\tilde{\alpha}_{1}\left(i d_{U_{\alpha}} \times \alpha_{2}\right)(u)$. Hence $E \circ \alpha=\tilde{\alpha}_{1} \circ\left(i d_{U_{\alpha}} \times \alpha_{2}\right)$ is smooth, as it is a composition of smooth maps.

We will now prove Theorem 3.2 in four steps. It is known that $\Psi$ is a bijection between $\operatorname{Maps}(X \times Y, Z)$ and $\operatorname{Maps}(X, \operatorname{Maps}(Y, Z))$.

So essentially we have the following situation: $\Psi$ is a bijection between sets $A$ and $B$ with inverse $\Phi$. To show that $\Psi$ induces a bijection of subsets $A^{\prime} \subset A$ and $B^{\prime} \subset B$, it suffices clearly to show $\Psi\left(A^{\prime}\right) \subset B^{\prime}$ and $\Phi\left(B^{\prime}\right) \subset A^{\prime}$. This is the purpose of the first two lemmas. Lemmas 3.7 and 3.8 finally show that $\Psi$ and $\Phi$ are smooth, and therefore morphisms in Diff. This concludes the proof of Theorem 3.2.

Lemma 3.5. If $f \in[X \times Y, Z]$, then $\bar{f} \in[X,[Y, Z]]$.

Proof. Let $f \in[X \times Y, Z]$. This means that for every plot $\left(\alpha_{1}, \alpha_{2}\right)$ for $X \times Y$, the map $f \circ\left(\alpha_{1}, \alpha_{2}\right)$ is a plot for $Z$.

We claim that $\bar{f} \in[X,[Y, Z]]$. First let us show that $\bar{f}$ takes values in $[Y, Z]$. To this end note that we can write $\bar{f}(x)$ as composition $f \circ \iota$ where $\iota: Y \rightarrow X \times Y$ maps $y$ to $(x, y)$. As the injection $\iota$ is smooth, so is the composition $\bar{f}$.

Now we prove smoothness of $\bar{f}$, that is, we show for every plot $\alpha \in \mathcal{P}(X)$ 
that $\bar{f} \circ \alpha \in \mathcal{P}([Y, Z])$. By the definition of the functional diffeology, this is true since

$$
\widetilde{\bar{f} \circ \alpha}:(u, y) \mapsto \bar{f}(\alpha(u))(y)=f(\alpha(u), y),
$$

so $\widetilde{\bar{f} \circ \alpha}=f \circ\left(\alpha \circ i d_{Y}\right)$ is a composition of smooth maps and therefore smooth.

Surjectivity of $\Psi$ follows from the next lemma.

Lemma 3.6. If $f \in[X,[Y, Z]]$ then $\tilde{f} \in[X \times Y, Z]$.

Proof. Let $f \in[X,[Y, Z]]$. Then we use the evaluation map to write

$$
\tilde{f}(x, y)=f(x)(y)=E(f(x), y) .
$$

So $\tilde{f}=E \circ\left(f \times i d_{Y}\right)$. By Lemma 3.4 the evaluation $E$ is smooth. Thus $\tilde{f}$ is a composition of smooth maps, and therefore smooth.

that for every $\left.\tilde{f} \circ \alpha \in \mathcal{P}(Z) .\left(\alpha_{2}(u)\right) \alpha_{2}(u)\right)$

Lemma 3.7. The map $\Psi:[X \times Y, Z] \rightarrow[X,[Y, Z]]$ is smooth.

Proof. We have to show that, given a plot $\alpha: U_{\alpha} \rightarrow[X \times Y, Z]$, the composition $\Psi \circ \alpha$ is a plot for $[X,[Y, Z]]$. This means that we have to show that

$$
\widetilde{\Psi \circ \alpha}: U_{\alpha} \times X \rightarrow[Y, Z]
$$

is smooth. Being smooth means that for every plot

$$
\beta=\left(\beta_{1}, \beta_{2}\right): U_{\beta} \rightarrow U_{\alpha} \times X,
$$

we get a plot $\widetilde{\Psi \circ} \alpha \circ \beta$ for $[Y, Z]$. We use the definitions to compute $\widetilde{\Psi \circ \alpha \circ}$ $\beta(u)=(\widetilde{\Psi \circ \alpha})\left(\beta_{1}(u), \beta_{2}(u)\right)$

$=\overline{\alpha\left(\beta_{1}(u)\right)}\left(\beta_{2}(u)\right) \in[Y, Z]$. This defines a plot if the map from $U_{\beta} \times Y \rightarrow Z$ given by $(\mathrm{u}, \mathrm{y}) \mapsto \overline{\alpha\left(\beta_{1}(u)\right)}\left(\beta_{2}(u)\right)(y)=\alpha\left(\beta_{1}(u)\right)\left(\beta_{2}(u), y\right)$ is smooth. To see that this is true, we use the hypothesis. By hypothesis, $\alpha: U_{\alpha} \rightarrow[X \times Y, Z]$ is a plot, so

$$
(v, x, y) \mapsto \alpha(v)(x, y)
$$

is smooth. But then the composition

$$
(u, y) \mapsto\left(\beta_{1}(u), \beta_{2}(u), y\right) \mapsto \alpha\left(\beta_{1}(u)\right)\left(\beta_{2}(u), y\right)
$$

is also smooth, and we are done. 
Lemma 3.8. The map $\Phi:[X,[Y, Z]] \rightarrow[X \times Y, Z]$ is smooth.

Proof. We have to show that given a plot $\alpha: U_{\alpha} \rightarrow[X,[Y, Z]]$, the map $\Phi \circ \alpha$ is a plot for $[X \times Y, Z]$. By definition of the functional diffeology this amounts to showing that

$$
(u, x, y) \mapsto \alpha(u)(x)(y)
$$

is smooth. Recall that smooth means that for each plot $\beta=\left(\beta_{1}, \beta_{2}, \beta_{3}\right)$ for $U_{\alpha} \times X \times Y$ we get a plot

$$
v \mapsto \alpha\left(\beta_{1}(v)\right)\left(\beta_{2}(v)\right)\left(\beta_{3}(v)\right)
$$

for Z.

We know that $\alpha$ is a plot, so again by definition of the functional diffeology, we know that $(u, x) \mapsto \alpha(u)(x) \in[Y, Z]$ is smooth. With $\beta$ being the plot from above, we get a plot $\left(\beta_{1}, \beta_{2}\right)$ for $U_{\alpha} \times X$, and hence a plot

$$
v \mapsto \alpha\left(\beta_{1}(v)\right)\left(\beta_{2}(v)\right)
$$

for $[Y, Z]$. Again we use the definition of functional diffeology to conclude that

$$
(v, y) \mapsto \alpha\left(\beta_{1}(v)\right)\left(\beta_{2}(v)\right)(y)
$$

is smooth. But now finally we use the plot $\left(, \beta_{3}\right): U_{\alpha} \rightarrow U_{\alpha} \times Y$ to conclude that

$$
v \mapsto \alpha\left(\beta_{1}(v)\right)\left(\beta_{2}(v)\right)\left(\beta_{3}(v)\right)
$$

is a plot for $Z$, which is what we had to show.

\section{Differential Forms}

It is not hard to generalize the notion of deRham differential forms to diffeological spaces. We will state the definition, and then show that, on manifolds, the definition coincides with the classical definition.

Definition 4.1 ( $\mathcal{D}$-forms). A $\mathcal{D}$-form of degree $n$ on a diffeological space $(X, \mathcal{P}(X))$ is a map $\omega$ which assigns to each plot $\alpha \in \mathcal{P}(X)$ a classical differential form $\omega(\alpha) \in \Omega^{n}\left(U_{\alpha}\right)$. We require the following compatibility condition: If $h \in \mathcal{L}^{p n}$ and $\alpha \in \mathcal{P}^{n}(X)$ are composable, then $\omega(\alpha \circ h)=$ $h^{*}(\omega(\alpha))=\omega(\alpha) \circ d h$. Let ${ }^{n}(X)$ denote the collection of all -forms of degree $n$ on $X$. 
Remark 4.2. Note that $\mathcal{D}^{n}(X)$ is a vector space if we define

$$
(s \omega+t \eta)(\alpha)=s \omega(\alpha)+t \eta(\alpha),
$$

given $\omega$ and $\eta$ in $\mathcal{D}^{n}(X)$ and scalars $s$ and $t$.

Next we want to show that this concept really generalizes differential forms, in the sense that if we apply this definition to manifolds, we get the classical differential forms.

Lemma 4.3. Given a smooth finite-dimensional manifold $M$, the vector space $\mathcal{D}^{n}(M)$ is isomorphic to $\Omega^{n}(M)$.

Proof. Given a $n$-form $\omega \in \Omega^{n}(M)$, we define a $\mathcal{D}$-form $\eta$ by $\eta(\alpha)=\alpha^{*}(\omega)$, the pullback of $\omega$ under the smooth map $\alpha$. As $\alpha^{*}: \Omega^{n}(M) \rightarrow \Omega^{n}\left(U_{\alpha}\right)$ is linear, this defines a linear map from $\Omega^{n}(M)$ into $\mathcal{D}^{n}(M)$. Now given a $\mathcal{D}$ form $\eta$ of degree $n$, we define $\omega \in \Omega^{n}(M)$ locally using charts. Given a chart $\phi: U \rightarrow V \subset \mathbf{R}^{k}$ for the manifold $M$, we define $\left.\omega\right|_{U}$ to be the pullback of $\eta\left(\phi^{-1}\right)$ under $\phi$ :

$$
\left.\omega\right|_{U}=\phi^{*}\left(\eta\left(\phi^{-1}\right)\right) .
$$

To see that this gives a well defined form on $M$, we have to see what happens if charts overlap. So let $\left(\phi_{1}, U_{1}\right)$ and $\left(\phi_{2}, U_{2}\right)$ be charts such that $U_{1} \cap U_{2}$ is nonempty. By definition of a smooth manifold, the change of coordinate map $h=\phi_{2} \circ \phi_{1}^{-1}$ is smooth. Therefore we can apply the compatibility condition in Definition 4.1 to get

$$
\eta\left(\phi_{1}^{-1}\right)=\eta\left(\phi_{2}^{-1} \circ h\right)=h^{*} \eta\left(\phi_{2}^{-1}\right)
$$

$=\left(\phi_{1}^{-1}\right)^{*} \phi_{2}^{*} \eta\left(\phi_{2}^{-1}\right)$.

Therefore

$$
\phi_{1}^{*} \eta\left(\phi_{1}^{-1}\right)=\phi_{2}^{*} \eta\left(\phi_{2}^{-1}\right)
$$

which shows that (4.1) defines a form on $M$. To see that (4.1) yields a linear map from $\mathcal{D}^{n}(M)$ to $\Omega^{n}(M)$ one uses the vector space structure from Remark 4.2 and the fact that $\phi^{*}$ is linear. The last step would be to show that the given maps are inverse to each other. This follows directly from the definitions, and we will omit the proof here.

Definition 4.4. Let 0 denote the subcategory of $\mathbf{O R}$ consisting of the objects which contain the origin, and the morphisms that map the origin to itself. Let $\mathcal{L}_{0}$ denote the collection of all morphisms in this category. Given a diffeology $\mathcal{P}$ on $X$, we denote by $\mathcal{P}_{x}$ the plots whose domains are in 0 and which map 0 to $x$. We will say that these plots are centered at $x$. 
Definition 4.5 (Value of a $\mathcal{D}$-form at a point). Given differential forms $\omega$ and $\eta$ on a diffeological space $(X, \mathcal{P})$ and a point $x \in X$, we say that $\omega$ and $\eta$ take the same value at $x$ and write $\omega \sim_{x} \eta$ if for all $\alpha \in \mathcal{P}_{x}$ we have

$$
\omega(\alpha)_{0}=\eta(\alpha)_{0} .
$$

The equivalence class of a form $\omega$ under $\sim_{x}$ is the value of $\omega$ at $x$, denoted by $\omega_{x}$.

Applications of differential forms on diffeological spaces include cohomology of path spaces ([Che86]) and quantization ([Sou85]).

\section{The Tangent Functor}

The following construction of the tangent functor is taken from [Hec95]. We present the construction in three steps. First we define the tangent space at a point, then the tangent bundle, and finally the differential of a smooth map. We conclude the chapter by discussing the relation between tangent vectors and $\mathcal{D}$-forms.

\subsection{Tangent Space at a Point}

Given throughout this section are a diffeological space $(X, \mathcal{P}(X))$ and a point $x \in X$. We want to construct a vector space $T_{x} X$ and linear maps $j_{\alpha}: T_{0} U_{\alpha} \rightarrow T_{x} X$ for each plot $\alpha$ centered at $x$. The maps $j_{\alpha}$ will be interpreted as differentials of the plots. The construction is motivated by the idea that those differentials satisfy a chain rule for plots of the form $\alpha \circ h$. Every choice of a plot $\alpha$ and a smooth map $h$ gives a reparametrization as in the following Figure 5.1.

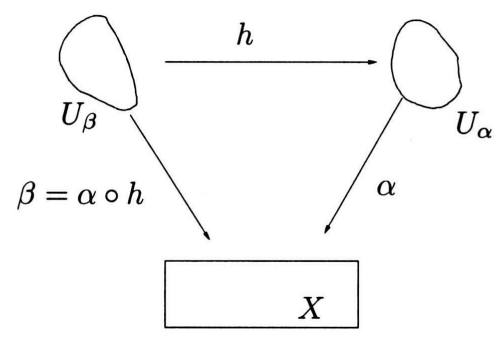

Figure 1. Reparametrization 
By 'chain rule' we mean that the corresponding diagram of differentials should commute:

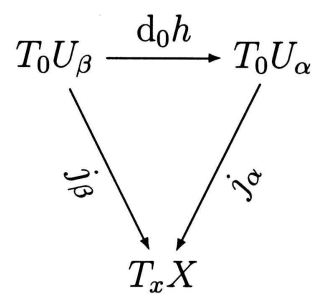

So we require

$$
j_{\alpha \circ h}=j_{\alpha} \circ d_{0} h
$$

which is the chain rule for the composition $\alpha \circ h$. This motivates the definition of the tangent space.

Definition 5.1 (Tangent Space). Let $E_{\alpha}$ denote the tangent space $T_{0} U_{\alpha}$. Let the direct sum

$$
E_{x}:=\bigoplus_{\alpha \in \mathcal{P}_{x}} E_{\alpha}
$$

be equipped with the final diffeology with respect to the injections

$$
\iota_{\alpha}: E_{\alpha} \rightarrow E_{x}
$$

(see Example 2.6). Often we will identify $v \in E_{\alpha}$ with its image under $\iota_{\alpha}$. Define a linear subspace of $E_{x}$ as follows.

$$
\left.\hat{E}_{x}:=\left\langle\iota_{\beta}(v)-\left(\iota_{\alpha} \circ d_{0} h\right)(v)\right| \beta=\alpha \circ h \text { and } v \in T_{0} U_{\beta}\right\rangle .
$$

Then the quotient space

$$
T_{x}(X):=E_{x} / \hat{E}_{x}
$$

is the tangent space to $X$ at $x$. If $\pi: E_{x} \rightarrow T_{x} X$ is the linear projection, we get a family $j_{\alpha}:=\pi \circ \iota_{\alpha}: E_{\alpha} \rightarrow T_{x} X$ of linear maps indexed by $\mathcal{P}_{x}$.

By construction, the tangent space together with the family $\left(j_{\alpha}\right)_{\alpha \in \mathcal{P}_{x}}$ is a colimit in the category of vector spaces. Thus we have a universal property. 
Lemma 5.2 (Universal Property). Assume that $F$ is a vector space together with a family of linear maps $\left(k_{\alpha}\right)_{\alpha \in \mathcal{P}_{x}}$ such that $k_{\alpha}: E_{\alpha} \rightarrow F$ and all the triangles

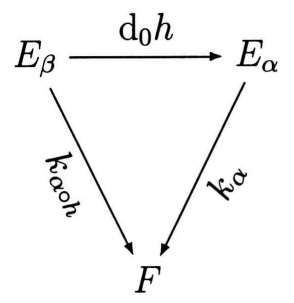

commute. Then there is a unique linear map $k: T_{x} X \rightarrow F$ such that for each $\alpha \in \mathcal{P}_{x}$ the triangle

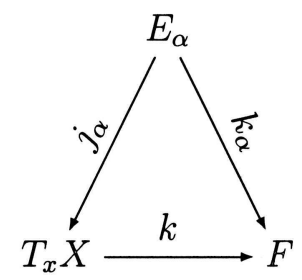

commutes.

Proof. First note that by the universal property for the direct sum $E_{x}$, the linear map

$$
\hat{k}:=\oplus_{\alpha \in \mathcal{P}_{x}} k_{\alpha}
$$

is the unique map such that

$$
k_{\alpha}=\hat{k} \circ j_{\alpha}
$$

for all $\alpha \in \mathcal{P}_{x}$. Using Diagram 5.1 we see that $\hat{k}$ vanishes on the subspace $\hat{E}_{x}$. Thus $\hat{k}$ factors through $T_{x} X$ : 


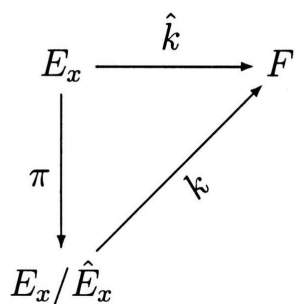

which yields existence of a map $k$ making the Diagrams 5.2 commute. To see that $k$ is unique, suppose that $k^{\prime}$ is another map making all Diagrams 5.2 commute. Note that the vector space $T_{x} X$ is generated by elements of the form $j_{\alpha}(v)$ with $v \in E_{\alpha}$. Commutativity of Diagram 5.2 yields

$$
k^{\prime}\left(j_{\alpha}(v)\right)=k(v) .
$$

So $k^{\prime}$ and $k$ agree on a set of vectors spanning $T_{x} X$, which proves $k^{\prime}=k$.

\subsection{Tangent Bundle}

We want to construct a new diffeological space, the tangent bundle of $X$. Just as in the classical case, the underlying set is the union of the tangent spaces to all points of $X$. The diffeology will be generated by the differentials of plots.

Definition 5.3. Let $T X$ be the disjoint union of all tangent spaces to $X$,

$$
T X:=\bigcup_{x \in X} T_{x} X
$$

Next we will define the differential of a plot, which will be a map $d \alpha$ : $T U_{\alpha} \rightarrow T X$.

Definition 5.4. Let $\alpha$ be a plot, not necessarily centered at a point $x \in X$. Given any point $u \in U_{\alpha}$, let

$h: \quad U_{\alpha} \rightarrow U_{\alpha}-u$

$$
y \mapsto y-u .
$$


Then we define the linear map

$$
d_{u} \alpha:=j_{\beta} \circ d_{u} h: T_{u} U_{\alpha} \rightarrow T_{\alpha(u)} X
$$

where $\beta=\alpha \circ h^{-1}$ is a plot centered at $\alpha(u)$. This yields a map

$$
d \alpha: T U_{\alpha} \rightarrow T X
$$

$$
v \mapsto d_{u} \alpha(v) \quad \text { if } v \in T_{u} U_{\alpha} .
$$

For $u=0$, the map $h$ is the identity map, and therefore this definition is consistent with letting $j_{\alpha}=d_{0} \alpha$ for plots centered at a point $x=\alpha(0)$.

Now we are ready to define a diffeology on $T X$.

Definition 5.5. Using $T U_{\alpha} \cong U_{\alpha} \times \mathbf{R}^{n}$ we can regard $T U_{\alpha}$ as an object in OR. So we can use the maps $d \alpha, \alpha \in \mathcal{P}(X)$ to generate a diffeology $\mathcal{P}(T X)$ on the set $T X$. The diffeological space $(T X, \mathcal{P}(T X))$ is the tangent bundle to the diffeological space $(X, \mathcal{P})$.

This is a natural definition and is similar to the manifold structure on the tangent bundle to a manifold. We want to see that the projection map, vector addition and scalar multiplication on $T X$ become smooth maps if $T X$ is equipped with this diffeology.

Lemma 5.6. The bundle projection $\pi: T X \rightarrow X$ is a smooth map.

Proof. By Lemma 1.14 it suffices to verify smoothness on a generating family, so we have to show that $\pi \circ d \alpha \in \mathcal{P}(X)$ for all plots $\alpha \in \mathcal{P}(X)$. But this follows immediately from the definitions, since $\pi \circ d \alpha=\alpha$.

Lemma 5.7. The scalar multiplication

$$
\begin{gathered}
\otimes: \mathbf{R} \times T X \rightarrow T X \\
(r, v) \mapsto r v
\end{gathered}
$$

is smooth. 
Proof. The diffeology of $\mathbf{R} \times T X$ is generated by $(\alpha, d \beta)$, where $\beta: U \rightarrow X$ is a plot and $\alpha: T U \rightarrow \mathbf{R}$ is a smooth map.

So let $(\alpha, d \beta)$ be a plot in the generating family. Then observe that

$$
\otimes \circ(\alpha, d \beta)(u)=\alpha(u) d \beta(u)=d \beta(\alpha(u) u)
$$

because of the linearity of each $d_{x} \beta$. Now define the map $\gamma: T U \rightarrow T U$ by $\gamma(u)=\alpha(u) u$.

The map $\alpha$ is smooth, and so is the scalar multiplication on $T U$, thus the map $\gamma$ is a smooth map, and we see that $\otimes \circ(\alpha, d \beta)=d \beta \circ \gamma$ is a plot for $T X$. This implies that $\otimes$ is a smooth map.

We have defined the functor $T$ on the objects of Diff. In the next subsection we will extend the definition to morphisms.

\subsection{Differentials of Smooth Maps}

Let us consider two spaces $(X, \mathcal{P}(X))$ and $(Y, \mathcal{P}(Y))$ and a smooth map $f \in[X, Y]$. We want to define a smooth map $T f: T X \rightarrow T Y$ by defining its restriction $T_{x} f$ to each fiber $T_{x} X$. So let $x \in X$ be any point and let $y=f(x)$. We will now use the Universal Property (Lemma 5.2) for $T_{x} X$ to define the map $T_{x} f: T_{x} X \rightarrow T_{y} Y$.

Definition 5.8. Consider the family

$$
\mathbf{F}=\left\{f \circ \alpha \mid \alpha \in \mathcal{P}_{x}(X)\right\} \subset \mathcal{P}_{y}(Y) .
$$

If $h \in \mathcal{L}_{0}$, then the map $f \circ \alpha \circ h$ is a plot for $Y$, centered at $y$. In particular we have that

$$
j_{f \circ \alpha \circ h}=j_{f \circ \alpha} \circ d_{0} h,
$$

so the family $\mathbf{F}$ makes the Diagram 5.1 commute. Hence by Lemma 5.2 there is a unique linear map

$$
T_{x} f: T_{x} X \rightarrow T_{y} Y
$$

such that for every $\alpha \in \mathcal{P}_{x}(X)$ we have

$$
d_{0}(f \circ \alpha)=T_{x} f \circ d_{0} \alpha .
$$


Remark 5.9. Note that we use different notation in order to distinguish differentials of plots $d \alpha: T U_{\alpha} \rightarrow T X$ and of smooth maps $T f: T X \rightarrow T Y$, because these two concepts are defined in a different way. However, we can regard $U_{\alpha}$ as a manifold and thus as a diffeological space, and in Chapter 6 we will prove the equality $d \alpha=T \alpha$ for plots.

Uniqueness of the differential $T_{x}$ yields the following chain rule.

Lemma 5.10 (Chain Rule). Given smooth maps $f$ and $g$, we have

$$
T_{x}(g \circ f)=T_{f(x)} g \circ T_{x} f .
$$

Proof. Looking at

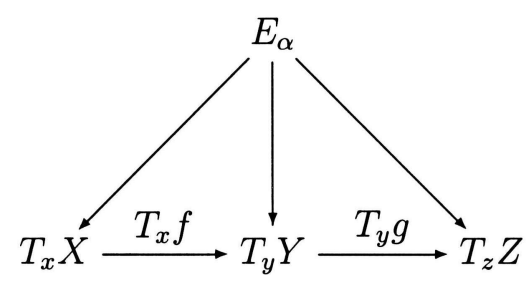

we see that the map $T_{f(x)} g \circ T_{x} g$ satisfies

$$
j_{g \circ f \circ \alpha}=T_{f(x)} g \circ T_{x} g \circ j_{\alpha} .
$$

But by Definition 5.8, $T_{x}(g \circ f)$ is the unique linear map with that property. Thus we have the equality

$$
T_{x}(g \circ f)=T_{f(x)} g \circ T_{x} g .
$$

Lemma 5.11 (Chain Rule for Plaques). Let $\alpha \in \mathcal{P}(X)$ and $f \in[X, Y]$. Then

$$
d(f \circ \alpha)=T f \circ d \alpha
$$


Proof. Given $u \in U_{\alpha}$, by definition of differential for plots we have

$$
d_{u}(f \circ \alpha)=d_{0}\left(f \circ \alpha \circ h^{-1}\right) \circ d_{u} h
$$

and

$$
T_{\alpha(u)} f \circ d_{u} \alpha=T_{\alpha(u)} f \circ d_{0}\left(\alpha \circ h^{-1}\right) \circ d_{u} h .
$$

We claim that the left hand sides are equal, so let us show that the right hand sides are equal. Let us write $\delta:=\alpha \circ h^{-1} \in \mathcal{P}(X)$. Then by Definition 5.8 we have that $d_{0}(f \circ \delta)=T_{\alpha(u)} f \circ d_{0} \delta$, which implies equality of the right hand sides.

Lemma 5.12. The map $T f: T X \rightarrow T Y$ is smooth.

Proof. By Lemma 1.14 it suffices to show

$$
T f \circ d \alpha \in \mathcal{P}(T Y)
$$

for all $\alpha \in \mathcal{P}(X)$. But this follows immediately from the chain rule for plots, since $T f \circ d \alpha=d(f \circ \alpha) \in \mathcal{P}(T Y)$.

Corollary 5.13. The assignments $X \mapsto T X$ and $f \mapsto T f$ define a functor

$$
T: \text { Diff } \rightarrow \text { Diff. }
$$

In Chapter 6 we will show that this functor extends the classical tangent functor for smooth manifolds.

\subsection{D-Forms and Tangent Vectors}

In this last subsection we want to show how 1-forms as defined in Chapter 4 yield smooth maps from $T X$ to $\mathbf{R}$.

Lemma 5.14. Let $\omega$ be a $\mathcal{D}$-form of degree 1 on the diffeological space $X$. The map $\omega_{x}: E_{x} \rightarrow \mathbf{R}$ defined by

$$
\omega_{x}\left(\sum_{\alpha} c_{\alpha} v_{\alpha}\right)=\sum_{\alpha} c_{\alpha} \omega_{x}\left(v_{\alpha}\right)
$$

$=\sum_{\alpha} c_{\alpha} \omega(\alpha)_{0}\left(v_{\alpha}\right)$ vanishes on $\hat{E}_{x}$ and therefore gives rise to a well-defined map

$$
\omega_{x}: T_{x} X \rightarrow \mathbf{R}
$$


Proof. It suffices to check that $\omega_{x}$ vanishes on vectors of the form

$$
\iota_{\beta}(v)-\left(\iota_{\alpha} \circ d_{0} h\right)(v)
$$

with $\beta=\alpha \circ h$ and $v \in E_{\beta}$. Omitting the $\iota$, we can reformulate this as

$$
\omega_{x}(v)=\omega_{x}\left(d_{0} h(v)\right) .
$$

The left hand side equals $\omega(\beta)_{0}(v)=\omega(\alpha \circ h)_{0}(v)$, which is the same as $h^{*}(\omega(\alpha))_{0}(v)$ by definition of $\mathcal{D}$-forms. The right hand side equals $\omega(\alpha)_{0}\left(d_{0} h(v)\right)=h^{*}(\omega(\alpha))_{0}(v)$ by definition of $h^{*}$.

Theorem 5.15. The map $\omega: T X \rightarrow \mathbf{R}$ sending $v \in T_{x} X$ to $\omega_{x}(v)$ is smooth if $\mathbf{R}$ is equipped with the manifold diffeology.

Proof. The diffeology of $T X$ is generated by the $d \alpha$, so it suffices to check that $\omega \circ d \alpha: T U_{\alpha} \rightarrow \mathbf{R}$ is a smooth map for each plot $\alpha \in \mathcal{P}(X)$. Given $v \in T_{u} U_{\alpha}$, we use the definition of $d \alpha$ to get

$$
\omega\left(d_{u} \alpha(v)\right)=\omega\left(j_{\beta}\left(d_{u} h(v)\right)\right)
$$

with $\beta=\alpha \circ h^{-1}$ and $h$ being the translation $y \mapsto y-u$. Let $w=d_{u} h(v)$. Then by definition of $\omega$ we get

$$
\begin{aligned}
& \omega\left(j_{\beta}(w)\right)=\omega_{\alpha(u)}\left(\pi\left(\iota_{\beta}(w)\right)\right) \\
= & \omega(\beta)_{0}(w) \\
= & \omega(\alpha)_{u}\left(d_{0} h^{-1}(w)\right) \\
= & \omega(\alpha)_{u}(v) \text { So } \omega \circ d \alpha=\omega(\alpha) \in \Omega^{1}\left(U_{\alpha}\right) \text { which proves that } v \mapsto \omega(d \alpha(v)) \text { is }
\end{aligned}
$$
smooth.

\section{The Tangent Functor on Manifolds}

The content of this section is also found in [Hec95]. We work out the proofs in some more detail.

We have seen in Lemma 2.1 that the category Mfd of smooth manifolds over $\mathbf{R}$ forms a full subcategory of Diff. Now we have two possibly different tangent functors on Mfd, the restriction of our diffeological tangent functor from Chapter 5, and the classical tangent functor. It is not clear however that the diffeological tangent bundle of a manifold is again a manifold.

The goal of this chapter is to show that the diffeological tangent functor 
indeed restricts to the category $\mathbf{M f d}$, and that this restriction agrees with the classical tangent functor. Until we prove equality, let us write $\tilde{T}$ for the diffeological tangent functor and $\tilde{d}$ for the differential of plots as defined in 5.4 .

We will have to show the following:

- The vector spaces $\tilde{T}_{x} M$ and $T_{x} M$ are isomorphic. This implies that the underlying sets of the tangent bundles are equal.

- The diffeology $\mathcal{P}(\tilde{T} M)$ defined in Chapter 5 is the same as the manifold diffeology consisting of all smooth maps into $T M$.

- $\tilde{T}_{x} f=T_{x} f$ for smooth maps $f \in C^{\infty}(M, N)=[M, N]$.

It then follows that $\tilde{T} f$ is smooth in the classical sense, because

$$
C^{\infty}(T M, T N)=[\tilde{T} M, \tilde{T} N] .
$$

We start by proving the first point:

Theorem 6.1. Let $M$ be a smooth manifold. For every point $x$ on $M$, the vector spaces $\tilde{T}_{x} M$ and $T_{x} M$ are isomorphic.

Proof. For each plot $\alpha$ centered at $x$ let

$$
k_{\alpha}:=d_{0} \alpha: E_{\alpha} \rightarrow T_{x} M,
$$

where $d_{0}$ denotes the classical differential. If $\beta=\alpha \circ h$ it follows from the classical chain rule that the triangle 5.1 commutes. So by Lemma 5.2 there is a unique linear map $k: \tilde{T}_{x} M \rightarrow T_{x} M$ such that $d_{0} \alpha=k \circ j_{\alpha}$ for each $\alpha \in \mathcal{P}_{x}(M)$. We claim that $k$ is an isomorphism. Let $(\psi, U)$ be a chart of $M$ about $x$ such that $\psi(x)=0$. Then $\phi:=\psi^{-1}$ is a plot centered at $x$, and $d_{0} \phi$ is a bijection. As $d_{0} \phi=k \circ j_{\phi}$, the map $k$ is necessarily surjective. It remains to show that $k$ is injective. In order to make the notation more readable we will identify $v_{\alpha} \in E_{\alpha}$ with its image under $\iota_{\alpha}$ in $E_{x}$, and we will write $[x]$ for the class of $x \in E_{x}$ in $T_{x} X$. So suppose

$$
0=\mathrm{k}\left(\left[\sum_{\alpha \in \mathcal{P}_{x}} c_{\alpha} v_{\alpha}\right]\right)
$$

$=\sum_{\alpha} c_{\alpha}\left(k \circ j_{\alpha}\right)\left(v_{\alpha}\right)$

$=\sum_{\alpha} c_{\alpha} d_{0} \alpha\left(v_{\alpha}\right)$ for some element $\sum_{\alpha} c_{\alpha} v_{\alpha} \in E_{x}$, where $v_{\alpha} \in E_{\alpha}$. Choose a chart $\psi$ as above, such that its inverse $\phi$ is a plot centered at $x$. Then

$$
d_{x} \psi: T_{x} M \rightarrow E_{\phi}
$$


is an isomorphism, and therefore $0=\mathrm{d}_{x} \psi\left(\sum_{\alpha} c_{\alpha} d_{0} \alpha\left(v_{\alpha}\right)\right)$

$=\sum_{\alpha} c_{\alpha} d_{0}(\psi \circ \alpha)\left(v_{\alpha}\right)$.

The vector $v_{\alpha}-d_{0}(\psi \circ \alpha)\left(v_{\alpha}\right)$ lies in $\hat{E}_{x}$ as $\alpha=\phi \circ(\psi \circ \alpha)$. So $\sum_{\alpha} c_{\alpha} v_{\alpha}=$ $\sum_{\alpha} c_{\alpha} v_{\alpha}-\sum_{\alpha} c_{\alpha} d_{0}(\psi \circ \alpha)\left(v_{\alpha}\right)$

$=\sum_{\alpha} c_{\alpha}\left(v_{\alpha}-d_{0}(\psi \circ \alpha)\left(v_{\alpha}\right)\right)$ represents the zero vector in $E_{x} / \hat{E}_{x}$, and therefore $k$ is injective.

To show the second point, we will first prove that the differential of plots as defined in 5.4 agrees with the classical differential.

Corollary 6.2. For a plot $\alpha$, the maps $\tilde{d} \alpha$ and $d \alpha$ agree.

Proof. We use the map $k$ from above Theorem 6.1 to identify diffeological and classical tangent space. So the equality

$$
k \circ j_{\alpha}=d_{0} \alpha,
$$

shows that $j_{\alpha}=\tilde{d}_{0} \alpha$ is to be identified with $d_{0} \alpha$. Now for $u \neq 0$ the map $\tilde{d}_{u} \alpha$ is defined as

$$
j_{\beta} \circ d_{u} h
$$

where $\beta=\alpha \circ h^{-1}$. Using $j_{\beta}=d_{0} \beta$ we get

$$
\tilde{d}_{u} \alpha=d_{0} \beta \circ d_{u} h=d_{u}(\beta \circ h)=d_{u} \alpha .
$$

Corollary 6.3. The manifold diffeology on $T M$ as defined in Subsection 2.1 and the tangent diffeology from Definition 5.5 are equal.

Proof. Recall from Definition 5.5 that the tangent diffeology on $T M$ is generated by the differentials $d \alpha$ of charts, which are smooth maps by Corollary 6.2. So the tangent diffeology is contained in the manifold diffeology, which consists of all smooth maps into $T M$.

On the other hand, by Lemma 2.3 the manifold diffeology is generated by $\mathbf{A}^{-1}$ where $\mathbf{A}$ is an atlas for the manifold. The tangent bundle has an atlas given by

$$
T \mathbf{A}:=\{d \phi \mid \phi \in \mathbf{A}\}
$$


so the inverse charts are of the form $d\left(\phi^{-1}\right)$. Now the smooth maps $\phi^{-1}$ are plots for $M$. Therefore the maps $d\left(\phi^{-1}\right)$ are contained in the generating set for the tangent diffeology. Hence the manifold diffeology is contained in the tangent diffeology. This implies equality of the two diffeologies.

Corollary 6.4. Given a smooth map $f: M \rightarrow N$ between smooth manifolds $M$ and $N$, the differentials $\tilde{T}_{x} f$ and $T_{x} f$ agree.

Proof. We have established the equality $j_{\alpha}=d_{0} \alpha$ for plots centered at $x$. Thus $j_{f \circ \alpha}=d_{0}(f \circ \alpha)=T_{x} f \circ d_{0} \alpha$ by the classical chain rule. But by construction, the diffeological differential $\tilde{T}_{x} f$ is the unique map satisfying this equality for all plots $\alpha \in \mathcal{P}_{x} X$. Thus $\tilde{T}_{x} f=T_{x} f$.

\section{Acknowledgements}

The author thanks his dissertation advisor Jimmie Lawson for making him aware of this interesting topic. The present text contains results of my research with Dr. Lawson since fall semester 2004.

\section{References}

[Che86] Kuo-Tsai Chen. On differentible Spaces, volume 1174: Categories in Continuum Physics of Lecture Notes in Mathematics, pages 38-42. Springer Verlag, (1986)

[Don86] Paul Donato. Geometrie des Orbites Coadjointes des Groupes de Diffeomorphismes, volume 1416 : Geometrie Symplectique et Mecanique, Coloque International, La Grande Motte of Lecture Notes in Mathematics, pp. 84-104, Springer Verlag, (1988)

[Frö86] A. Frölicher. Cartesian Closed Categories and Analysis of Smooth Maps, Volume 1174 : Categories in Continuum Physics of Lecture Notes in Mathematics, pp. 43-51, (1986).

[Gio03] P. Giordano. Infinitesimal Differential Geometry, Pre-print arXiv:math. DG/0308119v2 17 Dec 2003, arXiv, (2003). 
[Hec95] G. Hector. Géometrie et Topologie des Espaces Difféologiques, Analysis and Geometry in Foliated Manifolds, Proceedings of the VII International Colloquium on Differential Geometry, Santiago de Compostela, Spain, World Scientific, pp. 55-80, (1995).

[Mac71] Saunders Mc Lane. Categories for the Working Mathematician, Springer Verlag, New York, (1971).

[Sou85] Jean Marie Souriau. Un algorithme générateur de structures quantiques. Astérisque, hors série, Societé Mathémarique de France, pp. 341-399, (1985).

\author{
Martin Laubinger \\ Mathematics Department \\ Louisiana State University \\ Baton Rouge \\ Louisiana \\ e-mail : martinl@math.lsu.edu
}

